\title{
Varón de 30 años con hiperamonemia de 24 horas de evolución
}

\author{
30-year-old man with Acute Hyperammonemia
}

\begin{abstract}
Álvaro Hermida-Ameijeiras', Gemma Rodríguez Carnero², Miguel Ángel Martínez²
Unidad de Diagnóstico y Tratamiento de Enfermedades Metabólicas Congénitas. 'Servicio Medicina Interna. Estructura Organizativa de Gestión Integrada de Santiago. Área de Medicina de la Universidad de Santiago de Compostela. ${ }^{2}$ Servicio de Endocrinología y Nutrición. Estructura Organizativa de Gestión Integrada de Santiago
\end{abstract}

\section{RESUMEN}

La hiperamonemia es una de las complicaciones agudas más graves que se puede presentar en diversos trastornos congénitos del metabolismo como los defectos en el ciclo de la urea, las acidemias orgánicas o en las alteraciones en la oxidación de los ácidos grasos. Su reconocimiento y tratamiento es una emergencia clínica, dado que el efecto neurotóxico es directamente proporcional al tiempo de exposición a niveles elevados de amonio.

Presentamos el caso clínico de un paciente de 30 años con deterioro del nivel de consciencia y elevación del amonio plasmático de 24 horas de evolución.

Palabras clave: amonio, hiperamonemia, defectos de oxidación de ácidos grasos; hipoglucemia hipocetósica.

\section{CASO CLÍNICO}

Varón de 30 años que acude a Urgencias por deterioro del estado general secundario a ayuno, náuseas y vómitos de 24 horas de evolución.

Paciente sin hábitos tóxicos ni antecedentes alérgicos medicamentosos que refiere episodios de "migrañas" desde los 4 años de edad (aunque de mayor intensidad y más invalidantes desde los 25). Hasta la fecha había requerido tres ingresos hospitalarios previos (a los 5, 12 y 27 años) con un cortejo sintomático similar, en el que predominan los vómitos, la cefalea, somnolencia y desconexión medioambiental y que en su día se interpretaron como encefalitis vírica (tratado con aciclovir parenteral) en un caso y cefalea migrañosa en los otros dos, respectivamente. En ninguno de los ingresos citados, los estudios de neuroimagen objetivaron patología craneoencefálica evidenciable.

En la actualidad trabaja como fotógrafo y esporádicamente como camarero y el único tratamiento activo es un suplemento oral de vitamina B12 y ácido fólico.

El paciente relata a su llegada al Servicio de Urgencias que hace una semana comenzó con diarrea líquida con más de tres deposiciones diarias exentas de moco, pus o sangre y acompañadas de vómitos alimentarios. En los últimos tres días, además ha presentado rinorrea purulenta asociada a salvas de estornudos y tos con escasa expectoración. Por este motivo, el paciente se ha postrado en cama y disminuido la ingesta de sólidos y líquidos. La pareja con la que convive, lo trae a Urgencias alarmada por la creciente somnolencia y cefalea hemicraneal en las últimas 24 horas.

La exploración física en Urgencias mostraba taquicardia a 96 Ipm con el resto de constantes físicas en el rango de

\section{ABSTRACT}

Hyperammonemia is one of the most severe acute complication that can occur in various congenital metabolic conditions such as the urea cycle disorders, organic acidemias or fatty acids oxidation defects. The prompt recognition and treatment is a clinical emergency, since the neurotoxic effect is directly proportional to the time of exposure to high levels of ammonia.

We report the clinical case of a 30-year-old patient with a decreased level of consciousness and a 24-hour evolution of high plasma ammonia.

Keywords: Ammonia, hyperammonemia, fatty acid oxidation defects, hypoketotic hypoglycemia.

normalidad. La exploración física por aparatos, incluyendo la neurológica sistematizada, no mostraba anormalidad destacada salvo voz nasalizada secundaria a congestión nasal.

La analítica sanguínea al ingreso mostraba un pH de 7.4 con un exceso de bases efectivo de $-3.9 \mathrm{mEq} / \mathrm{L}$ y bicarbonato de $21.5 \mathrm{mmol} / \mathrm{L}$. Glucosa: $213 \mathrm{mg} / \mathrm{dL}$; creatinina: $0.8 \mathrm{mg} /$ dL; GOT (AST): 42 U/L; GPT (ALT): $60 \mathrm{U} / \mathrm{L} ; \mathrm{CPK}: 134 \mathrm{U} / \mathrm{L}$; amonio: $133.7 \mathrm{mmol} / \mathrm{L}$. La evolución durante el ingreso fue estable sin presentar datos de encefalopatía pero con valores crecientes de amonio plasmático hasta alcanzar los 181.8 $\mathrm{mmol} / \mathrm{L}$, motivo por el que se implantó tratamiento quelante de amonio con fenilbutirato sódico (4 grs cada 6 horas), suplementación oral con arginina y restricción de proteínas en la dieta, objetivando de esta manera una gran mejoría clínica y analítica. El estudio metabólico (que incluye acilcarnitinas/ carnitina libre plasmáticos, perfil de aminoácidos plasmáticos y determinación de ácidos orgánicos en orina) reflejaron un patrón de elevación de acilcarnitinas plasmáticas de cadena media y larga:

- Hexanoilcarnitina C6 $(0.35 \mu \mathrm{mol} / \mathrm{L} ; \mathrm{N}<0.32)$

- Octanoilcarnitina C8 (0.49 $\mu \mathrm{mol} / \mathrm{L} ; \mathrm{N}<0.26)$

- Decanoilcarnitina C10 (1.06 $\mu \mathrm{mol} / \mathrm{L} ; \mathrm{N}<0.33)$

- Decenoilcarnitina C10:1 (0.31 umol/L; $N<0.22)$

- Miristoleilcarnitina C14:1 (0.56 umol/L; $\mathrm{N}<0.47)$

- Miristodienoilcarnitina C14:2 (0.30 $\mu \mathrm{mol} / \mathrm{L} ; \mathrm{N}<0.11)$

- Palmitoleilcarnitina C16:1 (0.82 umol/L; $\mathrm{N}<0.39)$

Así como una disminución de carnitina libre $(11.24 \mu \mathrm{mol} / \mathrm{mmol}$ creat:; RN: 11.80-72.5) junto con una elevación de determinados ácidos orgánicos en orina (butirilglicina, isovalerilglicina, hexanoilglicina y heptenoilglicina) y disminución de otros (Ácido 
orótico y succinilacetona). El estudio de aminoácidos plasmáticos reflejaba niveles normales de glutamina y ligero descenso de arginina plasmática.

Con la sospecha bioquímica de trastorno de la betaoxidación de ácidos grasos y con niveles controlados de amonio plasmático y resolución sintomática, se decide suspender tratamiento con fenilbutirato e iniciar suplementación oral con riboflavina (50 mg/día) y carnitina (1800 mg/12 horas) así como ácido docosahexanoico y triglicéridos de cadena media (MCT).

De la misma manera, se solicitó cribado genético de variantes patogénicas en los genes ETFA, ETFB y ETFDH, detectándose la presencia de la variante [c.1601 C>T; p.Pro534Leu] en homocigosis en el exón 12 del gen ETFDH en el DNA de leucocitos del paciente, compatible con Déficit múltiple de acil-CoA deshidrogenasa (MADD) 0 aciduria glutárica tipo ॥ (OMIM 231680).

\section{DISCUSIÓN}

Los ácidos grasos constituyen una importante fuente de energía además de participar en la biosíntesis de macromoléculas. En situaciones de ayuno o de incremento de necesidades energéticas en las que se agotan las reservas de glucosa, muchos tejidos (excepto el cerebro) pueden utilizar los ácidos grasos para generar energía. Tanto el músculo esquelético y corazón, como el hígado dependen de la betaoxidación de los ácidos grasos para su correcto funcionamiento (de ahí que los trastornos de la betaoxidación cursen con afectación miocárdica, miopática y hepatocelular).

La oxidación de los ácidos grasos ocurre en la mitocondria para lo que se requiere la intermediación de un numeroso grupo de proteínas transportadoras y complejos enzimáticos con actividad acilCoAsintetasa que facilitan la captación de ácidos grasos de cadena larga y muy larga en las células y su conversión en acilésteres. Para alcanzar la matriz mitocondrial precisan del ciclo de la carnitina ${ }^{1}$.

En los períodos de incremento de demanda energética (ayuno prolongado, procesos infecciosos, intervenciones quirúrgicas, ejercicio físico intenso, ...) se requiere el suministro adicional procedente de la betaoxidación de los ácidos grasos y por ese motivo, en los trastornos de la betaoxidación se producen síntomas derivados del déficit energético a la par que la acumulación de los acilésteres genera toxicidad intracelular (las acilcarnitinas de cadena larga pueden producir arritmias cardíacas y afectación neurológica entre otras). Tanto la gluconeogénesis como la cetogénesis se encuentras inhibidas en caso de intoxicación lo que provoca episodios de hipoglucemia hipocetósica, academia láctica e hiperamoniemia, mientras que la inhibición del transporte de carnitina conlleva déficit de ésta en todos los trastornos de la betaoxidación ${ }^{1,2}$.

Los principales defectos de la betaoxidación son:

- Déficit de carnitina palmitoiltransferasa 1 (CPT 1) y 2 (CPT 2)

- Déficit del transportador de carnitina
- Déficit de acil-CoA deshidrogenasa de cadena corta (SCAD), media (MCAD) y muy larga (VLCAD)

- Déficit múltiple de acil CoA deshidrogenasa (MADD) 0 aciduria glutárica tipo II

- Déficit de L-3-hidroxiacil-CoA deshidrogenasa de cadena larga (LCHAD)

Todos estos trastornos siguen un patrón de herencia autosómica recesiva.

El déficit múltiple de acil-CoA deshidrogenasa también conocida como aciduria glutárica tipo II (GA-2; OMIM 231680), está causada por defectos en el transporte de electrones en el complejo mitocondrial ETF/ETF-Q0, causando un acúmulo intramitocondrial de acil-CoA ésteres. Las "flavoproteínas de transferencia de electrones" (ETF, por sus siglas en inglés) y "FTE-ubiquinona oxidoreductasa" (ETF: Q0, por sus siglas en inglés) ayudan a producir energía para descomponer ciertas grasas y proteínas de los alimentos que consumimos ${ }^{1-3}$.

Cuando alguna de estas dos enzimas falta, el cuerpo no puede descomponer las proteínas y la grasa para producir energía y debe utilizar glucosa. Aunque la glucosa es una buena fuente de energía, cuando se termina, el cuerpo trata de usar las grasas y las proteínas sin éxito. Esto lleva a una acumulación de ácido glutárico y otras sustancias tóxicas en la sangre.

La hipoglucemia puede hacer que un paciente se sienta débil, tembloroso o mareado y tenga la piel sudorosa y fría. La hipoglucemia puede suceder: después de hacer mucho ejercicio; después de comer demasiadas proteínas; después de períodos largos sin comer; durante enfermedades o infecciones. Los episodios de crisis metabólica pueden suceder por las mismas razones 4 .

La forma neonatal es la más severa y se acompaña además de los síntomas comunes a todos los trastornos de la betaoxidación (hipoglucemia hipocetósica, acidosis metabólica con anión gap elevado e hiperamoniemia), hepatomegalia, displasia renal quística y encefalopatía que puede causar el fallecimiento del neonato. Existe una forma infantil que aun siendo menos grave puede acarrear secuelas neuropsicológicas severas. La forma de debut en la edad adulta se considera más benigna y suele debutar con episodios de debilidad muscular (algunas personas con GA-2 nunca manifiestan síntomas y sólo se descubre que tienen la enfermedad después de habérsela diagnosticado a un hermano 0 hermana) ${ }^{2,5-6}$.

El estudio del plasma sanguíneo refleja hipoglucemias y en orina se objetiva elevación de ácidos orgánicos (ácido glutárico, 3-hidroxiglutárico, ácido glutacónico y glutaril carnitinas), junto con la deficiencia secundaria de carnitina libre?

La acidemia glutárica es un trastorno de origen genético. Todos tenemos un par de genes que produce la enzima ETF y otro par que produce la enzima ETF: Q0. El gen mutado se halla en el brazo corto (p) del cromosoma 19 (19p13.2). Dado que la MADD es un trastorno hereditario que se transmite por herencia autosómica recesiva, para padecer la enfermedad es necesario que se hallen alterados tanto el gen heredado 
del padre como el heredado de la madre. El estudio genético mostrará variantes patogénicas en los genes ETFA, ETFB y/0 ETFDH, estas últimas, respondedoras a riboflavina y vinculadas a la forma adulta como en el caso de nuestro paciente. La identificación del gen mutado nos permite además identificar a portadores y el diagnóstico prenatal ${ }^{8}$.

Los pacientes con MCADD necesitan comer con frecuencia para evitar la hipoglucemia 0 una crisis metabólica.

- Por lo general, se suele sugerir el consumo de una dieta rica en hidratos de carbono, usando hidratos de carbono de absorción lenta de forma fraccionada (pan, pastas, frutas, vegetales, etc.).

- Se deben restringir la ingesta de grasas y proteínas (carnes magras y alimentos lácteos bajos en grasa).

- Ante situaciones de estrés (infecciones, cuadros febriles) evitar ayuno prolongado asegurando una ingesta adecuada de hidratos de carbono (a base de bebidas o alimentos ricos en hidratos de carbono).

La mayoría de los niños no tiene crisis metabólicas después de los diez años. Sin embargo, algunos pueden necesitar continuar con el tratamiento de por vida. Conviene identificar precozmente los síntomas que pueden precipitar una crisis metabólica (falta de apetito, poca energía o demasiado sueño, vómitos, diarrea, una infección, fiebre).

Algunos adultos con GA-2 se benefician al tomar suplementos de riboflavina a diario (100-400 mg/d). A algunos pacientes se les puede dar L-carnitina para ayudarlos. Algunas personas con GA-2 se benefician al tomar suplementos de glicina. En aquellos pacientes con concentraciones disminuídas de CoQ10, está indicada su suplementación para mejorar la debilidad muscular 9 .

La detección precoz y las medidas de tratamiento precoces hacen que estos pacientes puedan llevar una vida normal y con muy buena evolución clínica.

\section{BIBLIOGRAFÍA}

1. Electron Transfer Flavoprotein-Ubiquinone Oxidoreductase: Glutaric Acidemia Type II. In: Scriver CR, Sly WS, Childs B, Beaudet AL, Valle D, Kinzler KW. The Metabolic and Molecular Bases of Inherited Disease. Vogelstein B; 2001

2. Goodman SI, Frerman FE: Glutaric acidaemia type II (multiple acyl-CoA dehydrogenation deficiency). J Inherit Metab Dis. 1984; 7(Suppl 1):33-37.

3. Amendt BA, Rhead WJ: The multiple acyl-coenzyme A dehydrogenation disorders, glutaric aciduria type II and ethylmalonic-adipic aciduria. Mitochondrial fatty acid oxidation, acyl-coenzyme A dehydrogenase, and electron transfer flavoprotein activities in fibroblasts. J Clin Invest. 1986; 78(1):205-213

4. Dusheiko G, Kew MC, Joffe BI, Lewin JR, Mantagos S, Tanaka K: Recurrent hypoglycemia associated with glutaric aciduria type II in an adult. N Engl J Med. 1979; 301(26):1405-1409.

5. Grice AS, Peck TE: Multiple acyl-CoA dehydrogenase deficiency: a rare cause of acidosis with an increased anion gap. Br J Anaesth. 2001; 86(3):437-441.

6. Ishii K, Komaki H, Ohkuma A, Nishino I, Nonaka I, Sasaki M: Central nervous system and muscle involvement in an adolescent patient with riboflavinresponsive multiple acyl-CoA dehydrogenase deficiency. Brain Dev. 2010; 32(8):669-672

7. Pollard LM, Williams NR, Espinoza L, Wood TC, Spector EB, Schroer RJ, et al. Diagnosis, treatment, and long-term outcomes of late-onset (type III) multiple acyl-CoA dehydrogenase deficiency. J Child Neurol. 2010; 25(8):954-960

8. Corydon MJ, Vockley J, Rinaldo P, Rhead WJ, Kjeldsen M, Winter V, et al. Role of common gene variations in the molecular pathogenesis of short-chain acyl-CoA dehydrogenase deficiency. Pediatr Res. 2001; 49(1):18-23.

9. Cotelli MS, Vielmi V, Rimoldi M, Rizzetto M, Castellotti B, Bertasi V, et al. Riboflavin-responsive multiple acyl-CoA dehydrogenase deficiency with unknown genetic defect. Neurol Sci. 2012; 33(6):1383-1387 